\title{
The Use of the Bethesda System for Reporting Thyroid Cytopathology in Korea: A Nationwide Multicenter Survey by the Korean Society of Endocrine Pathologists
}

Mimi Kim $\cdot$ Hyo Jin Park ${ }^{1} \cdot$ Hye Sook Min Hyeong Ju Kwon ${ }^{2,3}$. Chan Kwon Jung ${ }^{4}$ Seoung Wan Chae ${ }^{5}$. Hyun Ju Yoo ${ }^{6}$ Yoo Duk Choi ${ }^{7} \cdot \mathrm{Mi} \mathrm{Ja} \mathrm{Lee}^{8}$. Jeong Ja Kwak ${ }^{9}$ Dong Eun Song ${ }^{10} \cdot$ Dong Hoon Kim ${ }^{11}$ Hye Kyung Lee ${ }^{12}$. Ji Yeon Kim ${ }^{13}$ Sook Hee Hong ${ }^{14}$. Jang Sihn Sohn ${ }^{15}$ Hyun Seung Lee ${ }^{16}$. So Yeon Park ${ }^{1}$ Soon Won Hong ${ }^{17} \cdot$ Mi Kyung Shin ${ }^{18}$

Department of Pathology, Seoul National University Hospital, Seoul; 'Department of Pathology, Seoul National University Bundang Hospital, Seongnam; 'Department of Pathology, Severance Hospital, Yonsei University College of Medicine, Seoul; '3Department of Pathology, Wonju Severance Christian Hospital, Yonsei University Wonju College of Medicine, Wonju; ${ }^{4}$ Department of Hospital Pathology, College of Medicine, The Catholic University of Korea, Seoul; ${ }^{5}$ Department of Pathology, Kangbuk Samsung Hospital, Sungkyunkwan University School of Medicine, Seoul; ' Department of Pathology, Thyroid Center, Daerim St. Mary's Hospital, Seoul; ${ }^{7}$ Department of Pathology, Chonnam National University Medical School, Gwangju; ${ }^{8}$ Department of Pathology, Chosun University School of Medicine, Gwangju; 'Department of Pathology, Soon Chun Hyang University Hospital, Bucheon;

${ }^{10}$ Department of Pathology, Asan Medical Center, University of Ulsan College of Medicine, Seoul; ${ }^{11}$ Department of Pathology, Hallym University Sacred Heart Hospital,

Anyang: ${ }^{12}$ Department of Pathology, Eulji University School of Medicine, Daejeon; ${ }^{13}$ Department of Pathology, Inje University Haeundae Paik Hospital, Busan;

${ }^{14}$ Department of Pathology, Seegene Medical Foundation, Busan; ${ }^{15}$ Department of Pathology, Konyang University Hospital, Daejeon; ${ }^{16}$ Department of Pathology, Yangii General Hospital, Seoul; ${ }^{17}$ Department of Pathology, Thyroid Cancer Center, Gangnam Severance Hospital, Yonsei University College of Medicine, Seoul; ${ }^{18}$ Department of Pathology, Hallym University Kangnam Sacred Heart Hospital, Seoul, Korea

Received: January 6, 2017

Revised: April 3, 2017

Accepted: April 5, 2017

\section{Corresponding Author}

Mi Kyung Shin, MD, PhD

Department of Pathology, Hallym University

Kangnam Sacred Heart Hospital, Hallym University

College of Medicine, 1 Singil-ro, Yeongdeungpo-gu,

Seoul 07441, Korea

Tel: +82-2-2639-5114

Fax: +82-2-2633-7571

E-mail:smk0103@yahoo.co.kr

Hyo Jin Park, MD, PhD

Department of Pathology, Seoul National University

Bundang Hospital, 82 Gumi-ro 173beon-gil,

Bundang-gu, Seongnam 13620, Korea and Sheikh Khalifa

Specialty Hospital, P.O. Box 6365 Ras Al Khaimah, UAE

Tel: +971-7-201-2602

Fax: +82-31-787-4012

E-mail: hjparkmd92@gmail.com
Background: The Bethesda System for Reporting Thyroid Cytopathology (TBSRTC) has standardized the reporting of thyroid cytology specimens. The objective of the current study was to evaluate the nationwide usage of TBSRTC and assess the malignancy rates in each category of TBSRTC in Korea. Methods: Questionnaire surveys were used for data collection on the fine needle aspiration (FNA) of thyroid nodules at 74 institutes in 2012. The incidences and follow-up malignancy rates of each category diagnosed from January to December, 2011, in each institute were also collected and analyzed. Results: Sixty out of 74 institutes answering the surveys reported the results of thyroid FNA in accordance with TBSRTC. The average malignancy rates for resected cases in 15 institutes were as follows: nondiagnostic, 45.6\%; benign, 16.5\%; atypical of undetermined significance, 68.8\%; suspicious for follicular neoplasm (SFN), 30.2\%; suspicious for malignancy, $97.5 \%$; malignancy, $99.7 \%$. Conclusions: More than $80 \%$ of Korean institutes were using TBSRTC as of 2012. All malignancy rates other than the SFN and malignancy categories were higher than those reported by other countries. Therefore, the guidelines for treating patients with thyroid nodules in Korea should be revisited based on the malignancy rates reported in this study. 
The Bethesda System for Reporting Thyroid Cytopathology (TBSRTC) was developed to provide uniform terminology and diagnostic criteria for reporting thyroid fine needle aspiration (FNA) and to relate these cytologic diagnoses to clinical management. ${ }^{1}$ TBSRTC describes six categories for the diagnosis and reporting of thyroid FNAs, each with an assigned "risk of malignancy" and associated recommendations for clinical management. ${ }^{1}$ Since its implementation, many studies have demonstrated that TBSRTC has improved the quality of reporting by decreasing the number of ambiguous reports, increasing the positive predictive value of malignancy in thyroid glands that are operated, and decreasing the rates of surgery for benign thyroid nodules. ${ }^{2-5}$

TBSRTC terminology was incorporated into the 2009 guidelines of the Korean Thyroid Association (KTA) for management of patients with thyroid nodules and thyroid cancer. ${ }^{6}$ Several studies in Korea have demonstrated that TBSRTC well stratifies the malignancy risk by diagnostic categories and provides clinicians with useful information on the management of thyroid nodules. ${ }^{7-10}$ However, a wide variation in the incidence and malignant risk of each diagnostic category (DC; from here on, we will use DC as the abbreviation of diagnostic category), especially of the atypia of undetermined significance/follicular lesion of undetermined significance (AUS/FLUS) categories, has been reported. ${ }^{11,12}$ Moreover, the malignant risk of the categories has been reported to be higher than the implied risk of malignancy in TBSRTC. ${ }^{7,9,13-15}$

In the current study, we aimed to evaluate the extent of implementation of TBSRTC for reporting thyroid FNAs and its impact on managing patients with thyroid nodules by assessing the incidence and malignancy rate in each category of TBSRTC in Korea.

\section{MATERIALS AND METHODS}

\section{Design and data collection}

A questionnaire designed by the Endocrine Pathology Study Group of the Korean Society of Pathologists to gather data concerning the extent of implementation of TBSRTC and its impact on clinical practice was mailed to 211 pathology laboratories in the year 2012. Participants were asked questions related to the year of implementation of TBSRTC, number of thyroid aspiration cases in 2010 and 2011, usage of liquid-based cytology, types of liquid-based cytology used, and usage of core needle biopsy. Subsequently, a questionnaire was mailed to 41 institutions that had agreed to provide the statistics of thyroid FNA including the incidence, operation rate and malignancy rate of each diagnostic categories of TBSRTC. All the participants' answers were collated.
Among them, sixteen institutes reported the incidence of each category (unsatisfactory, benign, AUS, follicular neoplasm [FN], suspicious for malignancy [SM], and malignancy) in TBSRTC diagnosed from January to December, 2011. In total, 42,132 cases were analyzed. Follow-up malignancy rates of each category in 15 institutes were also collected and analyzed. Malignancy rate of each category was estimated based on surgically resected specimens. This survey was approved by the Institutional Review Board of Seoul National University Bundang Hospital, waiving the requirement for informed consent (IRB No. B-1212/182-304).

\section{Statistics}

The statistical significance of the data was assessed using SPSS ver. 21.0 for Windows (IBM Corp., Armonk, NY, USA). The sensitivity, specificity, positive predictive value, and negative predictive value were calculated in two different settings considering thyroid FNA as a "screening test." First, we calculated these parameters in malignant thyroid tumors. Surgical specimens which had been cytologically classified in the DC II of TBSRTC and diagnosed histologically as benign were considered to be truenegative samples. Surgical specimens whose cytologic diagnoses were in the remaining diagnostic categories (DC V, DC VI) and histologically proved to be malignant neoplasms were considered to be true-positives. The false-positive category included cases that had been diagnosed as SM or malignant (DC V, DC VI), but later confirmed histologically as benign (normal, non-neoplastic benign lesion, or benign neoplasm). The false-negative cases included those diagnosed as benign on FNA but confirmed as malignant upon surgical excision. In addition, those parameters were calculated for thyroid neoplasms including benign (such as follicular adenoma) and malignant. For this calculation, follow-up surgical specimens diagnosed as non-neoplastic benign disease or normal, which were interpreted as the DC II of TBSRTC, were considered to be true-negative samples, and the remaining diagnostic categories (DC IV, DC V, and DC VI), which proved to be either benign or malignant neoplasms by histologic examination of the surgical specimens, were considered to be true-positive. The false-positive category included cases that were diagnosed as FN, SM, and malignant but later confirmed histologically as benign non-neoplastic or normal. The false-negative cases included those diagnosed as DC II on FNA but confirmed as a true neoplasm upon surgical excision. The DC I and DC III (AUS/FLUS) categories were excluded from the statistical analysis because these diagnoses usually led to a repeat FNA rather than to surgical excision; moreover, these cases could not be categorized as either positive or negative. 


\section{RESULTS}

\section{General survey questions}

Questionnaire surveys were sent out to 77 institutes of pathology with controlled quality authorized by the Korean $\mathrm{Cy}$ topathology Conference. Three institutes were excluded because they were sending out their cytology specimens to consultants for the diagnosis, and answers from 74 institutes were included in the assessment. The survey results are summarized in Table 1.

Among the 74 institutes, 60 used TBSRTC for their diagnostic categorization of thyroid FNA at the time of survey. The starting year of using this classification varied from 2008 to 2012. The usage increased particularly from 2010 to 2012, 3 to 5 years after the conference in Bethesda. In Korea, the corresponding guidelines of the KTA for management of patients with thyroid nodules

Table 1. Summary of the survey

\begin{tabular}{|c|c|}
\hline & No. of institutions (\%) \\
\hline No. of institutions using TBSRTC & $60 / 74(81)$ \\
\hline The starting year using TBSRTC & 60 \\
\hline 2008 & 1 \\
\hline 2009 & 5 \\
\hline 2010 & 17 \\
\hline 2011 & 20 \\
\hline 2012 & 17 \\
\hline Methods of fine needle aspiration & 74 \\
\hline Conventional & 41 \\
\hline Liquid base & 11 \\
\hline Both & 22 \\
\hline The starting year using liquid based cytology & 33 \\
\hline 2000 & 1 \\
\hline 2002 & 1 \\
\hline 2006 & 1 \\
\hline 2007 & 2 \\
\hline 2008 & 5 \\
\hline 2009 & 5 \\
\hline 2010 & 10 \\
\hline 2011 & 3 \\
\hline 2012 & 3 \\
\hline Others & 2 \\
\hline Types of liquid based cytology & 33 \\
\hline Thin-prep & 16 \\
\hline Sure-path & 16 \\
\hline Others & 1 \\
\hline Main department performing FNA on patients & 74 \\
\hline Radiology & 38 (51.4) \\
\hline Pathology & $2(2.7)$ \\
\hline Internal medicine & $21(28.4)$ \\
\hline Surgery & $12(16.2)$ \\
\hline Thyroid clinician & $1(1.4)$ \\
\hline
\end{tabular}

TBSRTC, The Bethesda System for Reporting Thyroid Cytopathology; FNA, fine needle aspiration. and thyroid cancer were introduced in 2009, which might attribute in the subsequent sharp rise in the usage of TBSRTC. ${ }^{6,16}$ Forty-one out of the 74 institutes used conventional method for FNA, whereas 11 institutes used liquid-based method; the remaining 22 adopted both. Out of the 33 institutes using liquidbased preparation, 10 had started doing so in 2010. Regarding the preparation method for liquid-based FNA, two representative commercial methods, Thin-prep and Sure-path, were predominant: almost every institute chose one or the other method. Core needle biopsy was being done in 21 institutes (27\% of the 77). Among them, four institutes (5\% of the 77 ) used both core needle biopsy and FNA as the primary investigation method of thyroid nodules. In thirty-eight institutes (51.4\%), FNA was done by radiologists, followed by clinicians of internal medicine, surgeons, pathologists, and thyroid-specialized clinicians.

The survey indicated that the majority of the FNA specimens in Korea $(60 / 74,81 \%)$ were being diagnosed as per TBSRTC as of 2007. Moreover, the preparation method also shifted from conventional to liquid-based, the latter adopted in about $43 \%$ (33/77) including the institutes performing both.

\section{Incidence and malignancy rate of each DC of TBSRTC in Korea}

Sixteen out of 74 institutes answering the survey reported the results of thyroid FNA by TBSRTC. The mean distribution of six categories from 42,132 cases of 16 institutes was as follows: $11.1 \%$ nondiagnostic, $62.3 \%$ benign, $9.7 \%$ AUS, $0.9 \% \mathrm{FN}$, $6.7 \%$ SM, and $9.1 \%$ malignancy (Table 2). The mean operation rates of each category were as follows: $7.5 \%$ nondiagnostic, $2.4 \%$ benign, $20.2 \%$ AUS, $43.7 \%$ FN, $59.9 \%$ SM, and $69.2 \%$ malignancy (Table 3).

The average of malignancy rates for resected cases of 15 institutes are shown in Table 4: nondiagnostic, 45.6\%; benign, 16.5\%; AUS, $68.7 \%$; FN, 30.2\%; SM, 97.5\%; and malignancy, $99.7 \%$. Relatively high malignancy rates were noted in nondiagnostic and benign categories, which might have resulted from false-negative results of clinically suspicious nodules. Overall, the false-positive rate of the SM and malignancy category combined was $1.23 \%$.

\section{Follow-up histologic diagnosis of each categories of TBSRTC}

The final histological diagnoses upon resection are listed in Table 5. Each DC is sub-classified according to the recommendation of TBSRTC and follow-up histologic diagnoses are summarized by the sub-classification of each DC.

Of 4,599 cases, 247 were operated in the non-DC (DC I) and 
128 cases $(51.8 \%)$ were revealed as malignant nodules. The malignant cases mainly consisted of papillary thyroid carcinoma (121 cases), followed by five cases of follicular carcinoma, one case of poorly differentiated carcinoma, and one case of lymphoma. The benign category (DC II) had 422 cases of follow-up operation out of 21,399 cases. Among them, 320 (75.8\%) were revealed as benign nodules and 102 cases (24.2\%) were malignant: 89 papillary thyroid carcinomas, 12 follicular carcinomas, and one medullary carcinoma. Out of the 3,708 AUS-categorized cases, 722 had follow-up surgical resection (Table 3). The 514 cases (71.2\%) with malignant histologic diagnoses consisted of 471 cases of papillary carcinoma, 25 cases of follicular carcinoma, 13 of medullary carcinoma, four of Hurthle cell carcinoma, and one of poorly differentiated carcinoma. The remaining 208 (28.8\%) cases were proved to be benign lesions. In DC VI (FN), 121 out of 283 cases had surgical resection. Fortysix cases out of 121 cases (38\%) were malignant: 23 cases of follicular carcinoma, 20 of papillary carcinoma, and three of Hurthle cell carcinoma. The remaining cases ( 75 cases, 61.9\%) that were proved to be benign included 36 follicular adenomas, 19 nodular hyperplasia, 16 Hurthle cell adenomas, and four lymphocytic thyroiditis (Table 5). A total of 1,133 cases of DC V were histologically confirmed as benign nodules in 32 cases $(2.8 \%)$ and malignancy in 1,101 cases (97.2\%): 1,094 cases of papillary carcinoma and seven cases of medullary carcinoma (Table 5). Lastly, DC VI (malignancy) had 2,439 resected cases out of 3,343 FNAs. Overall, the final histologic diagnoses were well correlated with preoperative FNA diagnoses. Out of 2,439 cases, 2,419 (99.6\%) with a preoperative diagnosis of papillary carcinoma on FNA sub-classification were proved to be papillary carcinoma upon resection. Only 20 out of 2,439 resected cases were non-papillary thyroid carcinoma lesions including one follicular carcinoma, three medullary carcinomas, two poorly differentiated carcinomas, one undifferentiated carcinoma, one metastatic carcinoma, and 12 various benign lesions (Table 5).

\section{Diagnostic values for TBSRTC in Korea}

Through the data obtained by the survey summarized above, sensitivity, specificity, positive predictive value, and negative predictive value were analyzed in two ways. First, we included only those in the benign category (DC II) as negative and malignant

Table 2. The distribution of each diagnostic category of TBSRTC from 16 institutions

\begin{tabular}{|c|c|c|c|c|c|c|c|c|c|c|c|c|c|c|c|c|c|c|c|}
\hline & \multicolumn{16}{|c|}{ Diagnostic category } & \multirow{2}{*}{ otal } & \multirow{2}{*}{$\begin{array}{l}\text { Range } \\
(\%)\end{array}$} & \multirow{2}{*}{$\begin{array}{c}\text { Mean } \pm \text { SD } \\
(\%)\end{array}$} \\
\hline & 1 & 2 & 3 & 4 & 5 & 6 & 7 & 8 & 9 & 10 & 11 & 12 & 3 & 14 & 15 & 16 & & & \\
\hline$\sqrt{\mathrm{S} / \mathrm{I}}$ & $\begin{array}{l}1,040 \\
(14.7)\end{array}$ & $\begin{array}{r}386 \\
(21.2)\end{array}$ & $\begin{array}{r}865 \\
(16.6)\end{array}$ & $\begin{array}{r}199 \\
(10.7)\end{array}$ & $\begin{array}{r}513 \\
(11.7)\end{array}$ & $\begin{array}{r}185 \\
(10.0)\end{array}$ & $\begin{array}{l}285 \\
(7.5)\end{array}$ & $\begin{array}{r}64 \\
(8.0)\end{array}$ & $\begin{array}{r}315 \\
(17.2)\end{array}$ & $\begin{array}{r}400 \\
(13.1)\end{array}$ & $\begin{array}{l}154 \\
(6.8)\end{array}$ & $\begin{array}{r}208 \\
(10.4)\end{array}$ & $\begin{array}{r}522 \\
(16.9)\end{array}$ & $\begin{array}{r}39 \\
(6.4)\end{array}$ & $\begin{array}{r}7 \\
(0.9)\end{array}$ & $\begin{array}{r}97 \\
(5.8)\end{array}$ & $\begin{array}{l}5,279 \\
(12.5)\end{array}$ & 2 & 3 \\
\hline Benign & (47.9) & & & $\begin{array}{r}978 \\
(52.5)\end{array}$ & & $\begin{array}{l}1,099 \\
(59.7)\end{array}$ & & $\begin{array}{r}409 \\
(50.9)\end{array}$ & & & $\begin{array}{l}1,904 \\
(83.9)\end{array}$ & & & $\begin{array}{r}441 \\
(72.4)\end{array}$ & $\begin{array}{r}615 \\
(78.2)\end{array}$ & $\begin{array}{l}1,014 \\
(60.8)\end{array}$ & $\begin{array}{c}25,2364 \\
(59.9)\end{array}$ & & \\
\hline US & $\begin{array}{r}893 \\
(12.9)\end{array}$ & $\begin{array}{r}190 \\
(10.4)\end{array}$ & $\begin{array}{l}360 \\
(6.9)\end{array}$ & $\begin{array}{r}369 \\
(19.8)\end{array}$ & $\begin{array}{l}275 \\
(6.3)\end{array}$ & $\begin{array}{r}159 \\
(8.6)\end{array}$ & $\begin{array}{l}367 \\
(9.6)\end{array}$ & $\begin{array}{r}117 \\
(14.6)\end{array}$ & $\begin{array}{r}188 \\
(10.3)\end{array}$ & $\begin{array}{l}248 \\
(8.1)\end{array}$ & $\begin{array}{r}87 \\
(3.8)\end{array}$ & $\begin{array}{r}81 \\
(4.0)\end{array}$ & $\begin{array}{r}551 \\
(17.8)\end{array}$ & $\begin{array}{r}55 \\
(9.0)\end{array}$ & $\begin{array}{r}43 \\
(5.5)\end{array}$ & $\begin{array}{l}117 \\
(7.0)\end{array}$ & $\begin{array}{r}4,100 \\
(9.7)\end{array}$ & 9.8 & \\
\hline V/SFN & $\begin{array}{r}55 \\
(0.7)\end{array}$ & $\begin{array}{r}5 \\
(0.3)\end{array}$ & $\begin{array}{r}38 \\
(0.7)\end{array}$ & $\begin{array}{r}28 \\
(1.5)\end{array}$ & $\begin{array}{r}93 \\
(2.1)\end{array}$ & $\begin{array}{r}8 \\
(0.4)\end{array}$ & $\begin{array}{r}14 \\
(0.4)\end{array}$ & $\begin{array}{r}5 \\
(0.6)\end{array}$ & $\begin{array}{r}55 \\
(3.0)\end{array}$ & $\begin{array}{r}11 \\
(0.4)\end{array}$ & $\begin{array}{r}0 \\
(0)\end{array}$ & $\begin{array}{r}4 \\
(0.2)\end{array}$ & $\begin{array}{r}49 \\
(1.6)\end{array}$ & $\begin{array}{r}1 \\
(0.2)\end{array}$ & $\begin{array}{r}10 \\
(1.3)\end{array}$ & $\begin{array}{r}17 \\
(1.0)\end{array}$ & $\begin{array}{l}393 \\
(0.9)\end{array}$ & $0-2.1$ & 8 \\
\hline SFM & $\begin{array}{l}1,178 \\
(16.6)\end{array}$ & $\begin{array}{r}63 \\
(3.5)\end{array}$ & 269 & $\begin{array}{r}54 \\
(2.9)\end{array}$ & $\begin{array}{l}124 \\
(2.8)\end{array}$ & $\begin{array}{l}122 \\
(6.6)\end{array}$ & 204 & $\begin{array}{r}74 \\
(9.2)\end{array}$ & $\begin{array}{r}87 \\
(4.7)\end{array}$ & $\begin{array}{l}100 \\
(3.3)\end{array}$ & $\begin{array}{r}46 \\
(2.0)\end{array}$ & $\begin{array}{l}183 \\
(9.1)\end{array}$ & $\begin{array}{l}193 \\
(6.2)\end{array}$ & $\begin{array}{r}68 \\
(11.2)\end{array}$ & $\begin{array}{r}48 \\
(6.1)\end{array}$ & $\begin{array}{r}219 \\
(13.1)\end{array}$ & $\begin{array}{r}3,032 \\
(7.2)\end{array}$ & 2.0 & .1 \\
\hline aligna & $\begin{array}{l}521 \\
(7.4)\end{array}$ & $\begin{array}{l}133 \\
(7.3)\end{array}$ & $\begin{array}{r}889 \\
(17.1)\end{array}$ & $\begin{array}{r}236 \\
(12.7)\end{array}$ & $\begin{array}{r}546 \\
(12.5)\end{array}$ & $\begin{array}{r}269 \\
(14.6)\end{array}$ & $\begin{array}{l}329 \\
(8.6)\end{array}$ & $\begin{array}{r}135 \\
(16.8)\end{array}$ & $\begin{array}{l}102 \\
(5.6)\end{array}$ & $\begin{array}{r}73 \\
\quad(2.4)\end{array}$ & $\begin{array}{r}79 \\
(3.5)\end{array}$ & $\begin{array}{r}22 \\
(1.1)\end{array}$ & $\begin{array}{r}486 \\
(15.7)\end{array}$ & $\begin{array}{r}5 \\
(0.8)\end{array}$ & $\begin{array}{r}63 \\
(8.0)\end{array}$ & $\begin{array}{r}204 \\
(12.2)\end{array}$ & $\begin{array}{r}4,092 \\
(9.7)\end{array}$ & 7.1 & 5.5 \\
\hline Total & 7,084 & 1,822 & 5,212 & 1,864 & 4,376 & 1,842 & 3,810 & 804 & 1,834 & 3,050 & 2,270 & 2,010 & 3,091 & 609 & 786 & 1,668 & 42,132 & & \\
\hline
\end{tabular}

Values are presented as number (\%).

TBSRTC, The Bethesda System for Reporting Thyroid Cytopathology; SD, standard deviation; UNS, unsatisfactory; ND, non-diagnostic; AUS, atypia of undetermined significance; FN, follicular neoplasm; SFN, suspicious for follicular neoplasm; SFM, suspicious for malignancy.

Table 3. Operation rate by each diagnostic category of TBSRTC

\begin{tabular}{|c|c|c|c|c|c|c|c|c|c|c|c|c|c|c|c|c|}
\hline & \multicolumn{14}{|c|}{ Diagnostic category } & \multirow{2}{*}{ Range (\%) } & \multirow{2}{*}{ Mean \pm SD (\%) } \\
\hline & 1 & 2 & 3 & 4 & 6 & 7 & 8 & 9 & 10 & 11 & 12 & 13 & 14 & 15 & & \\
\hline UNS/ND & 1.6 & 6.5 & 10.2 & 2.5 & 3.2 & 5.3 & 42.2 & 1.9 & 0.5 & 3.9 & 2.9 & 10.2 & 0 & 14.3 & $0-42.2$ & $7.5 \pm 10.8$ \\
\hline Benign & 0.9 & 1.8 & 4.3 & 1.1 & 1.8 & 1.4 & 11.7 & 1.8 & 0.1 & 1.5 & 3 & 1.6 & 1.1 & 2.4 & $0.1-11.7$ & $2.4 \pm 2.8$ \\
\hline AUS & 10.5 & 23.2 & 28.1 & 9.5 & 16.4 & 38.7 & 23.9 & 16.5 & 13.3 & 13.8 & 25.9 & 24.5 & 5.5 & 32.6 & $5.5-38.7$ & $20.2 \pm 9.5$ \\
\hline FN/SFN & 45.5 & 60 & 71.1 & 32.1 & 37.5 & 35.7 & 100.0 & 29.1 & 54.5 & & 50 & 42.9 & 0 & 10 & $0-100$ & $43.7 \pm 25.6$ \\
\hline SFM & 9.9 & 63.5 & 82.5 & 90.7 & 63.9 & 74 & 75.7 & 46 & 75 & 23.9 & 59 & 67.9 & 25 & 81.3 & $9.9-90.7$ & $59.9 \pm 24.6$ \\
\hline Malignant & 52.2 & 72.9 & 86.5 & 69.5 & 73.2 & 73.3 & 90.4 & 54.9 & 80.8 & 39.2 & 68.2 & 72.8 & 40 & 95.2 & 40-95.2 & $69.2 \pm 17.2$ \\
\hline
\end{tabular}

Values are presented as percentage.

TBSRTC, The Bethesda System for Reporting Thyroid Cytopathology; SD, standard deviation; UNS, unsatisfactory; ND, non-diagnostic; AUS, atypia of undetermined significance; FN, follicular neoplasm; SFN, suspicious for follicular neoplasm; SFM, suspicious for malignancy. 
categories (DC V and VI) as positive. In this analysis, the sensitivity, specificity, positive predictive value and negative predictive value for malignant thyroid tumors were $97.19 \%, 87.91 \%$, $98.76 \%$, and $75.83 \%$, respectively. Second, we included FN (DC
IV) cases as positive and calculated each parameter for the neoplasm including both benign and malignant. In this case, the sensitivity, specificity, positive predictive value, and negative predictive value for neoplasm of thyroid were $97.14 \%, 72.89 \%$,

Table 4. The malignancy rate by each diagnostic category of TBSRTC

\begin{tabular}{|c|c|c|c|c|c|c|c|c|c|c|c|c|c|c|c|c|}
\hline & \multicolumn{14}{|c|}{ Diagnostic category } & \multirow{2}{*}{$\begin{array}{l}\text { Range } \\
(\%)\end{array}$} & \multirow{2}{*}{$\begin{array}{c}\text { Mean } \pm \text { SD } \\
(\%)\end{array}$} \\
\hline & 1 & 2 & 3 & 4 & 6 & 7 & 8 & 9 & 10 & 11 & 12 & 13 & 14 & 15 & & \\
\hline$\overline{V S / N D}$ & $\begin{array}{l}12 / 17 \\
(70.6)\end{array}$ & $\begin{array}{l}13 / 25 \\
(52)\end{array}$ & $\begin{array}{l}51 / 88 \\
(58)\end{array}$ & $\begin{array}{c}4 / 5 \\
(80)\end{array}$ & $\begin{array}{c}4 / 6 \\
(66.7)\end{array}$ & & $\begin{array}{r}4 / 27 \\
(14.8)\end{array}$ & $\begin{array}{l}0 / 6 \\
(0)\end{array}$ & $\begin{array}{l}0 / 2 \\
(0)\end{array}$ & $\begin{array}{c}2 / 6 \\
(33.3)\end{array}$ & $\begin{array}{l}0 / 6 \\
(0)\end{array}$ & & $\mathrm{NE}^{\mathrm{a}}$ & $\begin{array}{c}1 / 1 \\
(100)\end{array}$ & -100 & 2 \\
\hline Benign & $\begin{array}{l}10 / 32 \\
(31.3)\end{array}$ & $\begin{array}{r}2 / 19 \\
(10.5)\end{array}$ & $\begin{array}{l}43 / 119 \\
(36.1)\end{array}$ & $\begin{array}{r}2 / 11 \\
(18.2)\end{array}$ & $\begin{array}{l}1 / 20 \\
(5)\end{array}$ & & $5 / 48$ & & $\begin{array}{l}0 / 2 \\
(0)\end{array}$ & $\begin{array}{r}8 / 28 \\
(28.6)\end{array}$ & & $\begin{array}{l}0 / 20 \\
(0)\end{array}$ & $\begin{array}{l}0 / 5 \\
(0)\end{array}$ & & & \\
\hline AUS & $\begin{array}{l}53 / 94 \\
(56.4)\end{array}$ & $\begin{array}{l}33 / 44 \\
(75)\end{array}$ & & & & $\begin{array}{c}123 / 142 \\
(86.6)\end{array}$ & $\begin{array}{c}14 / 28 \\
(50)\end{array}$ & & & $\begin{array}{r}5 / 12 \\
(41.7)\end{array}$ & & & $\begin{array}{c}2 / 3 \\
(66.7)\end{array}$ & & & \\
\hline V/SFN & $\begin{array}{l}16 / 25 \\
(64)\end{array}$ & $\begin{array}{c}1 / 3 \\
(33.3)\end{array}$ & $\begin{array}{l}12 / 27 \\
(44.4)\end{array}$ & $\begin{array}{c}5 / 9 \\
(55.6)\end{array}$ & $\begin{array}{l}0 / 3 \\
(0)\end{array}$ & $\begin{array}{l}0 / 5 \\
(0)\end{array}$ & $\begin{array}{l}0 / 5 \\
(0)\end{array}$ & & $\begin{array}{l}0 / 6 \\
(0)\end{array}$ & $\mathrm{NE}^{\mathrm{a}}$ & $\begin{array}{l}0 / 2 \\
(0)\end{array}$ & $\begin{array}{r}3 / 19 \\
(15.8)\end{array}$ & $\mathrm{NE}^{\mathrm{a}}$ & $\begin{array}{c}1 / 1 \\
(100)\end{array}$ & 100 & $30.2 \pm 33.1$ \\
\hline SFM & $\begin{array}{c}115 / 117 \\
(98.3)\end{array}$ & $\begin{array}{l}40 / 40 \\
(100)\end{array}$ & $\begin{array}{c}213 / 222 \\
(95.9)\end{array}$ & $\begin{array}{c}49 / 49 \\
(100)\end{array}$ & $\begin{array}{l}72 / 78 \\
(92.3)\end{array}$ & $\begin{array}{c}148 / 151 \\
(98)\end{array}$ & $\begin{array}{l}47 / 56 \\
(83.9)\end{array}$ & $\begin{array}{l}40 / 40 \\
(100)\end{array}$ & $\begin{array}{l}74 / 75 \\
(98.7)\end{array}$ & $\begin{array}{l}11 / 11 \\
(100)\end{array}$ & $\begin{array}{c}106 / 108 \\
(98.1)\end{array}$ & $\begin{array}{c}\text { 3 131/131 } \\
(100)\end{array}$ & $\begin{array}{l}17 / 17 \\
(100)\end{array}$ & $\begin{array}{l}39 / 39 \\
(100)\end{array}$ & 00 & 5 \\
\hline aliana & $\begin{array}{c}\text { t } 271 / 272 \\
(99.6)\end{array}$ & $\begin{array}{l}97 / 97 \\
(100)\end{array}$ & $\begin{array}{c}\text { 760/769 } \\
(98.8)\end{array}$ & $\begin{array}{c}164 / 164 \\
(100)\end{array}$ & $\begin{array}{c}197 / 197 \\
(100)\end{array}$ & $\begin{array}{c}241 / 241 \\
(100)\end{array}$ & $\begin{array}{c}120 / 122 \\
(98.4)\end{array}$ & $\begin{array}{l}56 / 56 \\
(100)\end{array}$ & $\begin{array}{l}59 / 59 \\
(100)\end{array}$ & $\begin{array}{l}31 / 31 \\
(100)\end{array}$ & $\begin{array}{l}15 / 15 \\
(100)\end{array}$ & $\begin{array}{c}354 / 354 \\
(100)\end{array}$ & $\begin{array}{c}2 / 2 \\
(100)\end{array}$ & $\begin{array}{l}60 / 60 \\
(100)\end{array}$ & $98.4-100$ & $99.7 \pm 0.5$ \\
\hline
\end{tabular}

Values are presented as number (mal/op) (\%).

TBSRTC, The Bethesda System for Reporting Thyroid Cytopathology; SD, standard deviation; UNS, unsatisfactory; ND, non-diagnostic; AUS, atypia of undetermined significance; FN, follicular neoplasm; SFN, suspicious for follicular neoplasm; SFM, suspicious for malignancy.

${ }^{a}$ Not evaluated due to no case of operation.

Table 5. Follow up surgical diagnosis by each DC of TBSRTC

\begin{tabular}{|c|c|c|c|c|c|c|c|c|c|c|c|c|c|c|c|c|c|c|}
\hline \multirow{2}{*}{ DC } & & \multirow{2}{*}{$\begin{array}{l}\text { No. of } \\
\text { FNA }\end{array}$} & \multirow{2}{*}{$\begin{array}{c}\text { No. of } \\
\text { operation }\end{array}$} & \multicolumn{15}{|c|}{ Surgical diagnosis } \\
\hline & & & & PTC & FTC & HTC & MTC & PDC & UC & SCC & $\mathrm{MC}$ & Lym & FA & $\mathrm{HA}$ & $\mathrm{NH}$ & $\mathrm{LT}$ & GT & 0 \\
\hline \multirow[t]{3}{*}{ I } & Cyst & 796 & 35 & 7 & 1 & - & - & - & - & - & - & 1 & 3 & - & 22 & - & - & 1 \\
\hline & Acellular & 3,803 & 212 & 114 & 4 & - & - & 1 & - & - & - & - & 17 & 1 & 64 & 5 & 1 & 5 \\
\hline & Total & 4,599 & 247 & 121 & 5 & - & - & 1 & - & - & - & 1 & 20 & 1 & 86 & 5 & 1 & 6 \\
\hline \multirow[t]{5}{*}{$\|$} & $\mathrm{Be}$ & 19,179 & 375 & 73 & 11 & - & 1 & - & - & - & - & - & 34 & 2 & 240 & 7 & - & 7 \\
\hline & $\mathrm{LT}$ & 1,148 & 25 & 9 & - & - & - & - & - & - & - & - & - & - & - & 16 & - & - \\
\hline & GT & 49 & 0 & - & - & - & - & - & - & - & - & - & - & - & - & - & - & - \\
\hline & 0 & 1,023 & 22 & 7 & 1 & - & - & - & - & - & - & - & 4 & - & 9 & 1 & - & - \\
\hline & Total & 21,399 & 422 & 89 & 12 & - & 1 & - & - & - & - & - & 38 & 2 & 249 & 24 & - & 7 \\
\hline III & AUS & 3,708 & 722 & 471 & 25 & 4 & 13 & 1 & - & - & - & - & 52 & 13 & 124 & 11 & 1 & 7 \\
\hline IV & $\mathrm{FN}$ & 283 & 121 & 20 & 23 & 3 & - & - & - & - & - & - & 36 & 16 & 19 & 4 & - & - \\
\hline \multirow[t]{6}{*}{ V } & PTC & 1,715 & 1,123 & 1,093 & - & - & - & - & - & - & - & - & 1 & - & 19 & 10 & - & - \\
\hline & MTC & 11 & 8 & - & - & - & 7 & - & - & - & - & - & - & - & - & - & 1 & - \\
\hline & $M C$ & 2 & 0 & - & - & - & - & - & - & - & - & - & - & - & - & - & - & - \\
\hline & Lym & 1 & 0 & - & - & - & - & - & - & - & - & - & - & - & - & - & - & - \\
\hline & 0 & 12 & 2 & 1 & - & - & - & - & - & - & - & - & - & - & - & - & 1 & - \\
\hline & Total & 1,741 & 1,133 & 1,094 & - & - & 7 & - & - & - & - & - & 1 & - & 19 & 10 & 2 & - \\
\hline \multirow[t]{9}{*}{$\mathrm{Vl}$} & PTC & 3,313 & 2,429 & 2,419 & 1 & - & - & 1 & - & - & - & - & - & - & 4 & 4 & - & - \\
\hline & PDC & 2 & 2 & - & - & - & - & 1 & - & - & - & - & - & 1 & - & - & - & - \\
\hline & MTC & 5 & 3 & - & - & - & 3 & - & - & - & - & - & - & - & - & - & - & - \\
\hline & UC & 5 & 1 & - & - & - & - & - & 1 & - & - & - & - & - & - & - & - & - \\
\hline & SCC & 1 & 0 & - & - & - & - & - & - & - & - & - & - & - & - & - & - & - \\
\hline & $\mathrm{MC}$ & 8 & 3 & - & - & - & - & - & - & - & 1 & - & - & - & - & - & - & 2 \\
\hline & Lym & 2 & 0 & - & - & - & - & - & - & - & - & - & - & - & - & - & - & - \\
\hline & 0 & 7 & 1 & - & - & - & - & - & - & - & - & - & - & - & - & - & - & 1 \\
\hline & Total & 3,343 & 2,439 & 2,419 & 1 & - & 3 & 2 & 1 & - & 1 & - & - & 1 & 4 & 4 & - & 3 \\
\hline
\end{tabular}

DC, diagnostic category; TBSRTC, The Bethesda System for Reporting Thyroid Cytopathology; PTC, papillary thyroid carcinoma; FTC, follicular thyroid carcinoma; HTC, Hurthle cell thyroid carcinoma; MTC, medullary thyroid carcinoma; UC, undifferentiated carcinoma; SCC, squamous cell carcinoma; MC, metastatic carcinoma; Lym, Iymphoma; FA, follicular adenoma; HA, Hurthle cell adenoma; NH, nodular hyperplasia; LT, Iymphocytic thyroiditis; GT, granulomatous thyroiditis; O, others; Be, benign; AUS, atypia of undetermined significance; FN, follicular neoplasm; PDC, poorly differentiated carcinoma. 
$96.78 \%$, and $75.83 \%$, respectively.

\section{DISCUSSION}

TBSRTC was proposed in October 2007 in Bethesda, Maryland, and published in 2009,1 TBSRTC was then immediately introduced in the 2009 guidelines of the KTA., ${ }^{6,16}$ Despite the information about TBSRTC having been globally disseminated, the status of its usage in clinical practice in Korea has been unknown. This questionnaire provides a cross-sectional view, based on the collective data from participating pathology laboratories, of the degree of implementation of TBSRTC in Korea and evaluates its impact on daily practice by assessing the incidence and malignant risk of each DC at each pathology laboratory using TBSRTC.

Overall, the data shows that TBSRTC is well implemented in Korea. As of July 2012 (when the questionnaire was mailed), $81 \%$ (60 out of 74) of the participating pathology laboratories were already using TBSRTC, which is higher than the rate given in published data from the College of American Pathologists. ${ }^{17}$ In addition, the data demonstrates interesting technical points. Fifty-seven percent of the laboratories are using conventional alcohol-fixed direct smears as a preparatory method for thyroid FNA; in contrast, $14.9 \%$ of the laboratories are using liquidbased cytology and $29.7 \%$ are using both. Overall, $44.6 \%$ of the laboratories are using liquid based cytology. Twenty-one out of 74 pathology laboratories (27\%) received thyroid core needle biopsies; among them, four institutes used both core needle biopsy and FNA as the primary diagnostic test for thyroid nodules. Although core needle biopsy of thyroid nodules is emerging as an effective alternative method, the primary use of core needle biopsy in a thyroid nodule is quite unusual. For example, current American Thyroid Association thyroid nodule management guidelines and the Society of Radiologists in Ultrasound Consensus Statement have not included core biopsy as an evaluation tool of thyroid nodule, and some other studies suggest that after several non-diagnostic FNA, surgical excision should be considered appropriate. ${ }^{18,19}$

More importantly, our data showed some differences in the incidence and malignant risk of certain categories between TBSRTC1 or published studies from other countries ${ }^{7,12}$ and the current results. As shown in Table 2, lower incidence of DC IV (0.9 $\pm 0.8 \%)$ and higher incidence of DC III $(9.7 \pm 4.6 \%), \mathrm{DC}$ V $(6.7 \pm$ $4.1 \%)$, and DC VI $(9.1 \pm 5.5 \%)$ were demonstrated. Moreover, the malignancy rates of DC I, II, III, and V (mean of malignancy rates: $46.5 \pm 33.5,16.5 \pm 13.1,68.7 \pm 15.8$, and $97.5 \pm 4.5$, re- spectively) in our study were higher than the risk of malignancy indicated by the original TBSRTC (1\%-4\%, DC I; $0 \%-3 \%$, DC II; 5\%-15\%, DC III, and 60\%-75\%, DC V) ${ }^{1}$ and other previous studies. ${ }^{11,18-24}$ However, these findings are consistently shown in previous reports from Korea. ${ }^{8,9}$ These can be explained by several reasons. First, in Korea, the prevalence of papillary thyroid carcinoma is more than $95 \%$, whereas that of follicular carcinoma is very low, at 3.2\%, ${ }^{6}$ which has resulted in the lower incidence of DC IV and higher incidence of DC III, V, and VI. In addition, higher prevalence of papillary thyroid carcinoma might influence higher malignant rates of DC II, DC III, and DC V. Second, most institutes submitting their details of TBSRTC are universitybased tertiary centers. In tertiary referral institutes, the patients who had been diagnosed with suspicious or malignant nodules at primary care centers were more likely to receive an operation despite a DC III or DC V diagnosis of FNA, especially those whose imaging studies strongly suggest malignancy. In the present study, malignancy rates were calculated based on the resected cases. The malignancy rate assessed either in the total fine needle aspiration cytology cases or in the resected cases cannot be said to represent the exact malignancy rate of each DC of TBSRTC. The former can miss unresected malignant tumors, and the latter can be exaggerated by selection bias, which may occur in this type of retrospective study. Therefore, our malignancy rates cannot be directly compared with the malignant risk recommended in TBSRTC. Lastly, because Korean citizens tend to consider false-positive results more significantly than false-negative results, cytopathologists in Korea may have a tendency to under-diagnose FNA of thyroid nodules. ${ }^{10}$

There are some limitations in the data derived from this questionnaire, especially those evaluating the incidence and malignancy rate of each DC in TBSRTC, as mentioned above. First, 14 out of 16 institutes submitting the incidence and malignancy rate of TBSRTC are university-based centers. Therefore, the results of this survey may not be truly representative of all kinds of practices; from community to academic. Second, all data are self-reported and not verified. Third, the data might not reflect the current status in certain aspects because the questionnaire was mailed in July 2012. The implementation of TBSRTC is more widespread in Korea at present, and liquid-based cytology and core needle biopsy are likely to have been adopted more widely. The usage of core needle biopsy in thyroid nodules has been actively studied in Korea. ${ }^{25}$

In conclusion, overall, TBSRTC has been well adopted in Korea, with more than $80 \%$ of institutes using TBSRTC as of 2012. However, ongoing education is still necessary to reduce 
the variation of incidence and malignant rates of TBSRTC. Moreover, it would be better to modify reference values of malignancy rate of each category of TBSRTC and revisit the guidelines for treating patients with thyroid nodules in Korea based on the results of this study. Finally, it is highly recommended that each institute review their report of thyroid aspiration and have their own incidence and malignant rates for each DC.

\section{Conflicts of Interest}

No potential conflict of interest relevant to this article was reported.

\section{Acknowledgments}

This research was supported by The Korean Society for Cytopathologists Grant No. 2012-01.

\section{REFERENCES}

1. Cibas ES, Ali SZ; NCI Thyroid FNA State of the Science Conference. The Bethesda System For Reporting Thyroid Cytopathology. Am J Clin Pathol 2009; 132: 658-65.

2. Bongiovanni M, Krane JF, Cibas ES, Faquin WC. The atypical thyroid fine-needle aspiration: past, present, and future. Cancer Cytopathol 2012; 120: 73-86.

3. Crowe A, Linder A, Hameed O, et al. The impact of implementation of the Bethesda System for Reporting Thyroid Cytopathology on the quality of reporting, "risk" of malignancy, surgical rate, and rate of frozen sections requested for thyroid lesions. Cancer Cytopathol 2011; 119: 315-21.

4. Ozluk Y, Pehlivan E, Gulluoglu MG, et al. The use of the Bethesda terminology in thyroid fine-needle aspiration results in a lower rate of surgery for nonmalignant nodules: a report from a reference center in Turkey. Int J Surg Pathol 2011; 19: 761-71.

5. Horne MJ, Chhieng DC, Theoharis C, et al. Thyroid follicular lesion of undetermined significance: Evaluation of the risk of malignancy using the two-tier sub-classification. Diagn Cytopathol 2012; 40: 410-5.

6. Yi KH, Park YJ, Koong SS, et al. Revised Korean Thyroid Association management guidelines for patients with thyroid nodules and thyroid cancer. Endocrinol Metab 2010; 25: 270-97.

7. Kim SK, Hwang TS, Yoo YB, et al. Surgical results of thyroid nodules according to a management guideline based on the BRAF (V600E) mutation status. J Clin Endocrinol Metab 2011; 96: 658-64.

8. Lee YB, Cho YY, Jang JY, et al. Current status and diagnostic values of the Bethesda system for reporting thyroid cytopathology in a papillary thyroid carcinoma-prevalent area. Head Neck 2017; 39: 269-74.

9. Park JH, Yoon SO, Son EJ, Kim HM, Nahm JH, Hong S. Incidence and malignancy rates of diagnoses in the bethesda system for reporting thyroid aspiration cytology: an institutional experience. Korean J Pathol 2014; 48: 133-9.

10. Lee K, Jung CK, Lee KY, Bae JS, Lim DJ, Jung SL. Application of Bethesda system for reporting thyroid aspiration cytology. Korean J Pathol 2010; 44: 521-7.

11. Bongiovanni M, Spitale A, Faquin WC, Mazzucchelli L, Baloch ZW. The Bethesda System for Reporting Thyroid Cytopathology: a meta-analysis. Acta Cytol 2012; 56: 333-9.

12. Ohori NP, Schoedel KE. Variability in the atypia of undetermined significance/follicular lesion of undetermined significance diagnosis in the Bethesda System for Reporting Thyroid Cytopathology: sources and recommendations. Acta Cytol 2011; 55: 492-8.

13. Mathur A, Najafian A, Schneider EB, Zeiger MA, Olson MT. Malignancy risk and reproducibility associated with atypia of undetermined significance on thyroid cytology. Surgery 2014; 156: 1471-6.

14. Ho AS, Sarti EE, Jain KS, et al. Malignancy rate in thyroid nodules classified as Bethesda category III (AUS/FLUS). Thyroid 2014; 24: 832-9.

15. VanderLaan PA, Marqusee E, Krane JF. Clinical outcome for atypia of undetermined significance in thyroid fine-needle aspirations: should repeated fna be the preferred initial approach? Am J Clin Pathol 2011; 135: 770-5.

16. Yi KH, Park YJ, Koong SS, et al. Revised Korean Thyroid Association Management Guidelines for patients with thyroid nodules and thyroid cancer. Korean J Otorhinolaryngol-Head Neck Surg 2011; 54 : 8-36.

17. Auger M, Nayar R, Khalbuss WE, et al. Implementation of the Bethesda System for Reporting Thyroid Cytopathology: observations from the 2011 thyroid supplemental questionnaire of the College of American Pathologists. Arch Pathol Lab Med 2013; 137: 1555-9.

18. Haugen BR, Alexander EK, Bible KC, et al. 2015 American Thyroid Association management guidelines for adult patients with thyroid nodules and differentiated thyroid cancer: The American Thyroid Association Guidelines Task Force on Thyroid Nodules and Differentiated Thyroid Cancer. Thyroid 2016; 26: 1-133.

19. Samir AE, Vij A, Seale MK, et al. Ultrasound-guided percutaneous thyroid nodule core biopsy: clinical utility in patients with prior nondiagnostic fine-needle aspirate. Thyroid 2012; 22: 461-7.

20. Yang J, Schnadig V, Logrono R, Wasserman PG. Fine-needle aspiration of thyroid nodules: a study of 4703 patients with histologic and clinical correlations. Cancer 2007; 111: 306-15.

21. Yassa L, Cibas ES, Benson CB, et al. Long-term assessment of a mul- 
tidisciplinary approach to thyroid nodule diagnostic evaluation. Cancer 2007; 111: 508-16.

22. Mufti ST, Molah R. The bethesda system for reporting thyroid cytopathology: a five-year retrospective review of one center experience. Int J Health Sci (Qassim) 2012; 6: 159-73.

23. Baloch ZW, Hendreen S, Gupta PK, et al. Interinstitutional review of thyroid fine-needle aspirations: impact on clinical management of thyroid nodules. Diagn Cytopathol 2001; 25: 231-4.
24. Wu HH, Rose C, Elsheikh TM. The Bethesda system for reporting thyroid cytopathology: An experience of 1,382 cases in a community practice setting with the implication for risk of neoplasm and risk of malignancy. Diagn Cytopathol 2012; 40: 399-403.

25. Yi KS, Kim JH, Na DG, et al. Usefulness of core needle biopsy for thyroid nodules with macrocalcifications: comparison with fineneedle aspiration. Thyroid 2015; 25: 657-64. 